\title{
Resolved magic-angle spinning of anisotropic samples in Inhomogeneous Fields
}

\author{
Carlos A. Meriles, Dimitris Sakellariou and Alexander Pines* \\ Materials Sciences Division, Lawrence Berkeley National Laboratory \\ and Department of Chemistry, University of California \\ Berkeley, CA 94720, USA
}

\begin{abstract}
The chemical shift spectrum of a liquid embedded in a porous sample spinning at the magic angle has been recovered in the presence of static field and radio-frequency gradients. Field inhomogeneity and susceptibility broadening are averaged by a procedure that combines magic-angle turning with a train of $z$-rotation pulses. The experiment emulates the situation encountered in "ex-situ" NMR in which the sample is located away from the field sources. Given the equivalence of field and sample spinning, the results suggest that the use of a rotating magnetic field and refocusing pulses might enable the study of samples such as solids or fluids in porous materials external to the magnet.
\end{abstract}




\section{Introduction}

During the last two decades a variety of increasingly demanding applications has led to the development of the so-called "inside-out" or "ex-situ" NMR, in which the sample under study sits outside the bore of the magnet. ${ }^{1,2,3,4}$ Although strong field inhomogeneities seem to hinder NMR spectroscopy, a potential approach to recover chemical shift information under this geometry, has been recently suggested. ${ }^{5}$ The method relies on the use of a composite $z$-rotation pulse train inducing nutation echoes in the presence of matched $\mathrm{B}_{0}$ and $\mathrm{B}_{1}$ gradients. In principle, the matching can be achieved geometrically (by designing the shape of the field sources) or actively (by crafting appropriate matching pulses).

Such approaches do not, however, remove the broadening due to anisotropic interactions such as magnetic susceptibility, chemical shift anisotropy and dipolar couplings. The standard tool to remove these broadening mechanisms is to average them out by sample spinning. For "ex-situ" situations where the sample may be required to remain static, rotating the magnetic field at the magic angle may be more appropriate. In fact, this idea was suggested by Andrew and Eades ${ }^{6}$ and used later in pulsed mode to perform coherent averaging in zero-field spectroscopy. ${ }^{7}$ At high fields, the implementation of rotating magnetic fields has not been attempted because the power required to switch the field direction becomes enormous at spinning rates beyond several kHz. In this communication, we demonstrate an approach to the possibility of high resolution NMR of anisotropic samples in inhomogeneous fields by combining correlated magnetic and rf fields with magic-angle spinning to remove, at the same time, line broadening due to field inhomogeneities and anisotropic interactions. The principle is 
shown to work well in an experiment with simulated conditions in which a porous rock sample spins at a low rate $(200 \mathrm{~Hz})$ while being exposed to combined $\mathrm{rf}$ and field gradients. Despite susceptibility broadening and field inhomogeneity, the isotropic chemical shift resolved spectrum of the liquid inside the pores can be recovered.

\section{Pulsing scheme}

For samples rotating under homogenous magnetic fields, several schemes have been developed to suppress the sidebands ${ }^{8}$, some of them being particularly well suited for low spinning rates. ${ }^{9,10}$ Magic Angle Turning ${ }^{11}$ (MAT) is one such case, consisting of a preparation pulse followed by five $\pi$ pulses synchronized with the rotation period. The pulse timing on the $\pi$ pulses is selected so that a sideband-free spectrum containing the isotropic chemical shift information is obtained in the indirect dimension.

If during the free evolution period in between the $\pi$ pulses, the spin ensemble dephases in strong field gradients (as in "ex-situ" applications), the resulting spectrum still can be severely broadened. In this case, $z$-rotation pulses can be applied to refocus inhomogeneities by inducing nutation echoes whose amplitudes keep track of the dephasing induced by chemical shift differences during the free evolution period. The $\pi$ pulses can be merged into a $z$-rotation pulse train by simply reversing the (overall) phase of one of the (composite) $\pi / 2$ sandwich pulses. Accordingly, a MAT experiment can be run in the presence of strong inhomogeneities by applying the same train with phases of the five $\pi / 2$ pulses changed during the evolution period as depicted in Fig.1. 


\section{Experiments}

As a proof of principle as well as a measure of its potential usefulness, the above procedure was used in an experiment involving slow spinning in the presence of magnetic inhomogeneity. As shown in Fig. (2), a sedimentary rock (Berea sandstone) initially saturated with water was spun at $\sim 200 \mathrm{~Hz}$ about an axis at the magic angle. The sample was located outside of the rf coil and exposed to a field gradient roughly matched to the $\mathrm{rf}$ inhomogeneity. The ${ }^{1} \mathrm{H}$ NMR spectrum of the absorbed water inside the rock when the field is homogenous is shown in Fig. (3a). Trace amounts of paramagnetic metals on the walls of the pores result in a significant susceptibility mismatch between the rock and the fluid. ${ }^{12}$ The resulting nonuniform magnetic field in the pore space broadens the NMR resonance. This anisotropic interaction is inhomogeneous causing the spectrum to break into narrow sidebands even at very low spinning speeds. Rotational echoes disappear as a strong field gradient $(\sim 8 \mathrm{kHz} / \mathrm{cm})$ is applied (Fig. (3b)). However, most of the original spectral features can be recovered if a train of $z$-rotation pulses periodically refocuses the dephasing induced by field inhomogeneities: as shown in Fig. (3c), stroboscopic sampling of the nutation echoes restores most of the hidden sideband pattern.

The low spinning rate attainable still represents a serious limitation for NMR spectroscopy of more complex fluids. This situation can be addressed by the use of a modified MAT pulse sequence. Fig. (4) shows the two-dimensional spectrum obtained after replacing the water content of the rock by pure ethanol. Again the sample spins at $\sim 200 \mathrm{~Hz}$ while being exposed to a field gradient of $\sim 8 \mathrm{kHz} / \mathrm{cm}$ and, as before, the direct dimension consists of an overlapping sideband pattern, where no resonances can be 
identified. Upon application of the pulse scheme of Fig. 1b, an extra modulation is imposed that provides the sideband-free isotropic chemical shift resolved spectrum along the indirect dimension. The attainable resolution of $\sim 1 \mathrm{ppm}$ is enhanced in comparison to Fig. (3b) in which field inhomogeneity and susceptibility mismatch lead to an overall broadening of $\sim 10 \mathrm{ppm}$.

\section{Conclusions}

Discerning oil from water contained in rocks at high depths by means of an "inside-out" probe represents an obvious and exciting goal of the proposed methodology. However, applications are wider and include in-vivo spectroscopy, often compromised by susceptibility related problems. Furthermore, with sufficient rf power spin decoupling and Hartmann-Hahn cross-polarization transfer appear to be possible, thereby opening the way to the possibility of "ex-situ" NMR spectroscopy on solids.

Practical difficulties to overcome in a real "ex-situ" setup are still very important and are mainly linked to three main sources: the first one, mentioned previously, ${ }^{5}$ relates to the performance of the pulse train in the presence of strong gradients. Offset imperfections are inadequately compensated for, the current scheme relying on the rf amplitude and appropriate selection of the tuning frequency. This obviously represents a serious restriction to be overcome by the development of more robust pulse trains. The second problem arises from the fact that the magnetic fields for external samples are necessarily much lower than those available inside the bore of the magnet. As the average field strength diminishes, the sensitivity decays making detection considerably more difficult. Pre-polarizing the samples or using other nuclei as external sources of 
polarization are possible solutions. Finally, because very strong currents are required to induce even moderate external fields, spinning rates in excess of hundreds of $\mathrm{Hz}$ will be difficult to achieve. These challenges are being undertaken and experiments involving exsitu NMR spectroscopy and magic angle field spinning are currently on their ways in our laboratory.

\section{Aknowledgment}

The authors want to thank Y. -Q. Song for generously providing the porous rock samples. This work was supported by the Director, Office of Science, Office of Basic Energy Sciences, Materials Sciences Division, of the U.S. Department of Energy under Contract No. DE-AC03-76SF00098. 


\section{Figure captions}

Fig. 1: (a) Basic MAT pulse timing. After initial excitation, five $\pi$ pulses are consecutively applied during a fixed time period equal to an integer number of rotations $n T$ (multiples of three are not allowed). ${ }^{11}$ In its simplest version, the second and the fourth pulses are held at $n T / 3$ and $2 n T / 3$ respectively while the first, third and fifth pulses sweep along the intermediate time intervals. For a given separation of the pulses, direct acquisition is made immediately after $n T$. (b) In the presence of matched $\mathrm{rf}$ and field gradients, inhomogeneities can be periodically refocused by a train of composite $z$ rotation pulses. Stroboscopic acquisition at the induced (nutation) echo maxima provides an FID free of field inhomogeneities while keeping the chemical information. ${ }^{5} \pi$ pulses can be easily merged into the train by simply reversing the phase in one of the $\pi / 2$-pulses. If $n T$ / 6 equals exactly an integer number of train periods, an MAT-like experiment can be performed by selectively reversing the overall phase on five of the (composite) $\pi / 2$ pulses as shown. The latter condition, easily achievable in the rotating field case, can be satisfied in general by adjusting the free evolution interval and the duration $\beta$.

Fig. 2: Experimental setup used to simulated the "ex-situ" magic angle field spinning. A sedimentary rock (Berea sandstone) shaped as a cylinder $(\varnothing=12 \mathrm{~mm}, \mathrm{~h}=12 \mathrm{~mm})$ spins at the magic angle $\left(54.7^{\circ}\right)$ at about $200 \mathrm{~Hz}$. The rock sits close to the edge but outside the rf coil used for excitation and detection and inside a 15-mm zirconia rotor. A pair of Maxwell coils induces an adjustable field gradient along the $B_{1}$ axis, not along the

rotation axis as it first seems. ${ }^{13}$ The homemade probe head rests inside a superwide bore 
4.2 T magnet operated by a Varian/Chemagnetics Infinity spectrometer. All distances are given in $\mathrm{mm}$.

Fig. 3: 1D NMR proton spectra of a porous rock saturated with water and spinning at 221 Hz. (a) With no gradient applied and inside a fairly homogeneous field, the proton spectrum displays a broadening, of the order of $15 \mathrm{ppm}(2.7 \mathrm{kHz})$, induced by paramagnetic impurities on the walls of the pores. Because this anisotropic interaction is inhomogeneous, the profile breaks into narrow sidebands even at low spinning rates as recorded after a single $\pi / 2$ pulse excitation ( 8 scans). (b) Same experiment but in the presence of a gradient of $8 \mathrm{kHz} / \mathrm{cm}$, resulting in a broadening of the line profile (32 scans). (c) Even in this gradient, rotational echoes (and, with them, the original shape of the spectrum) are almost completely recovered by means of a train of refocusing composite $z$-rotation pulses. The series $(2 \gamma)_{97.2}(4 \gamma)_{291.5}(2 \gamma)_{97.2}(\gamma)_{0}$ was used as a composite $\pi / 2$ pulse $^{14}$. The nominal $90^{\circ}$ length $\gamma$ was $5 \mu$ s and the free evolution period was $47 \mu \mathrm{s} ; \beta$ was $17 \mu$ s and the number of rotor periods $n$ was 2 .

Fig. 4: $2 \mathrm{D}$ proton spectrum of pure ethanol contained in the rock after running a modified MAT experiment shown in Fig. 1. The gradient along the sample was $8 \mathrm{kHz} / \mathrm{cm}$ and the spinning speed was $214 \mathrm{~Hz}$. The direct dimension in which the slow spinning sideband spectrum appears is plotted horizontally: due to strong overlapping; no clear indication of ethanol can be discerned from the spectrum. The indirect dimension, displaying the isotropic chemical shifts, is plotted vertically and, despite some residual broadening, the 
expected pattern can be clearly recognized. As a reference, the resolution of $\sim 1 \mathrm{ppm}$ can be compared with that of Fig. 3b, $\sim 10 \mathrm{ppm}$. 
Figure 1: Meriles et al.

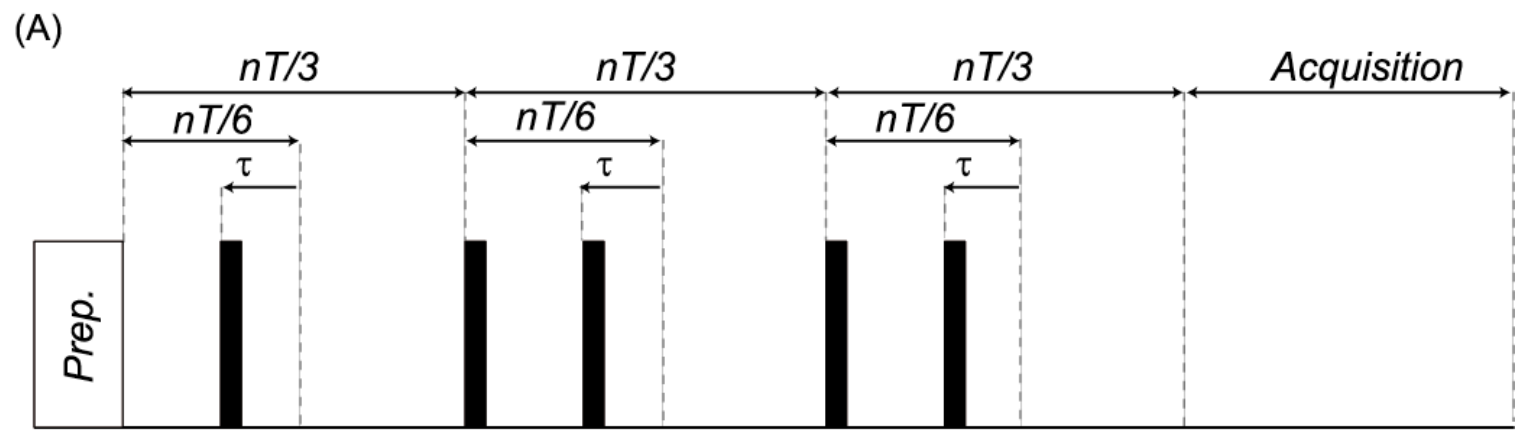

(B)
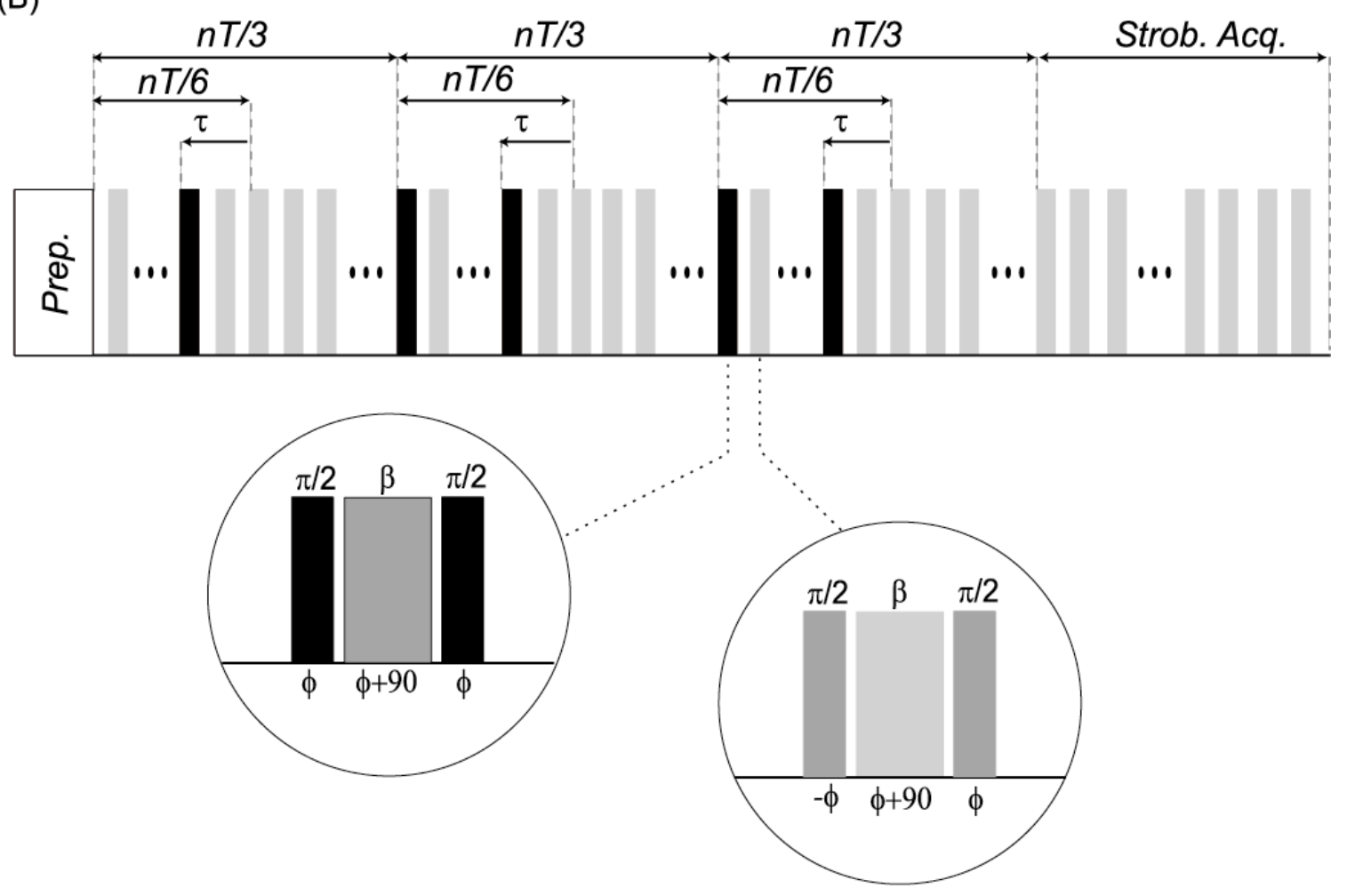
Figure 2: Meriles et al.

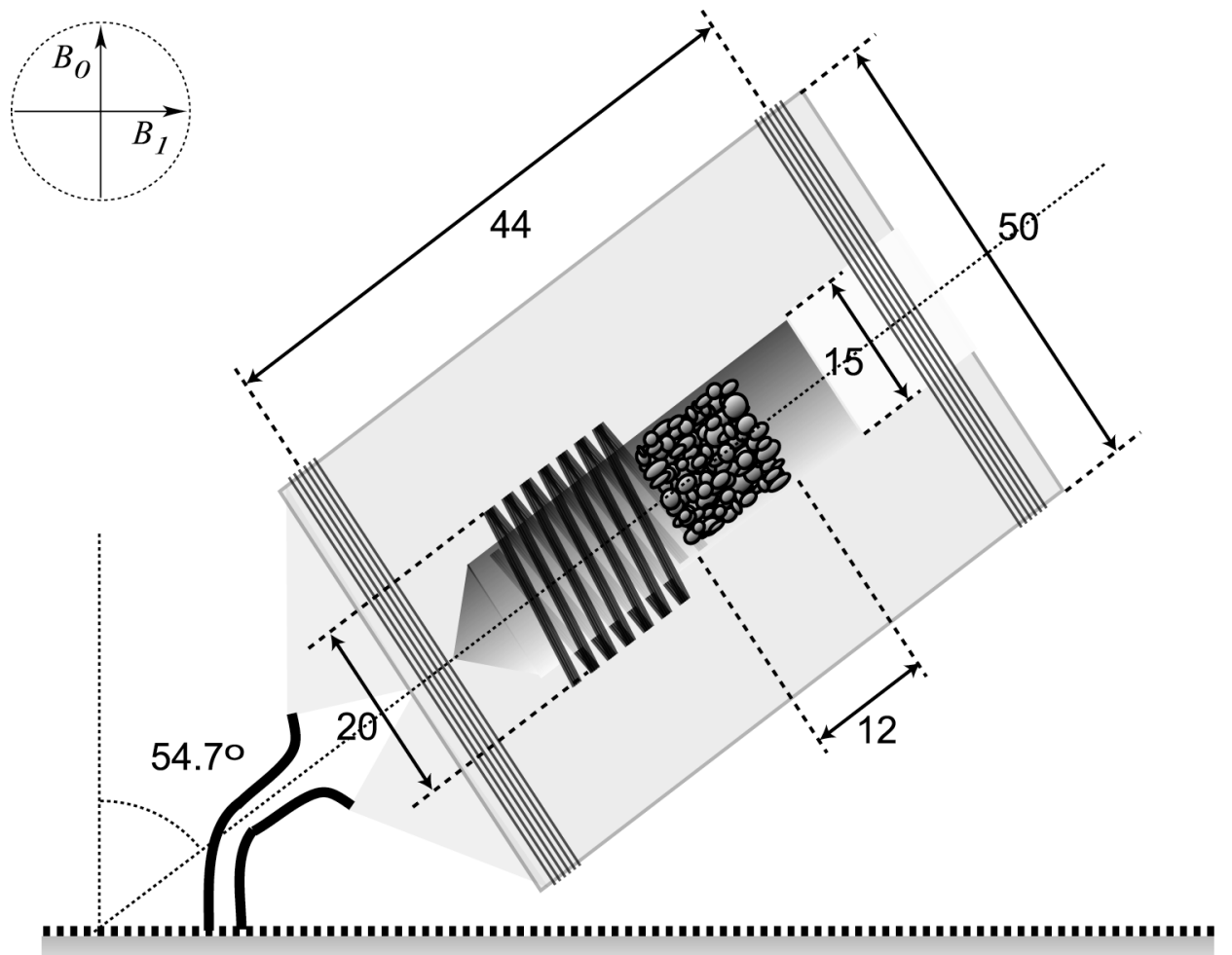


Figure 3: Meriles et al.

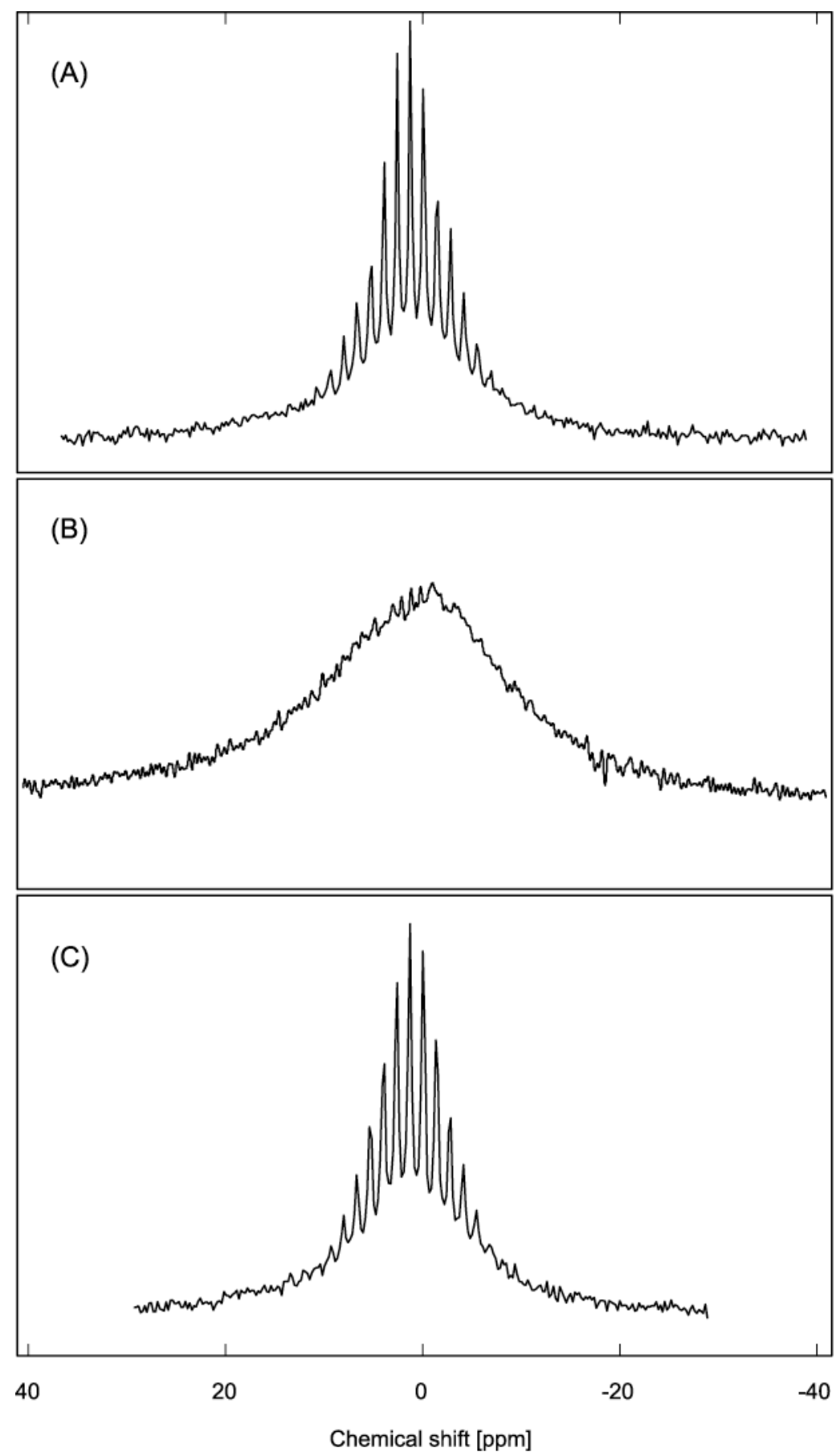


Figure 4: Meriles et al.

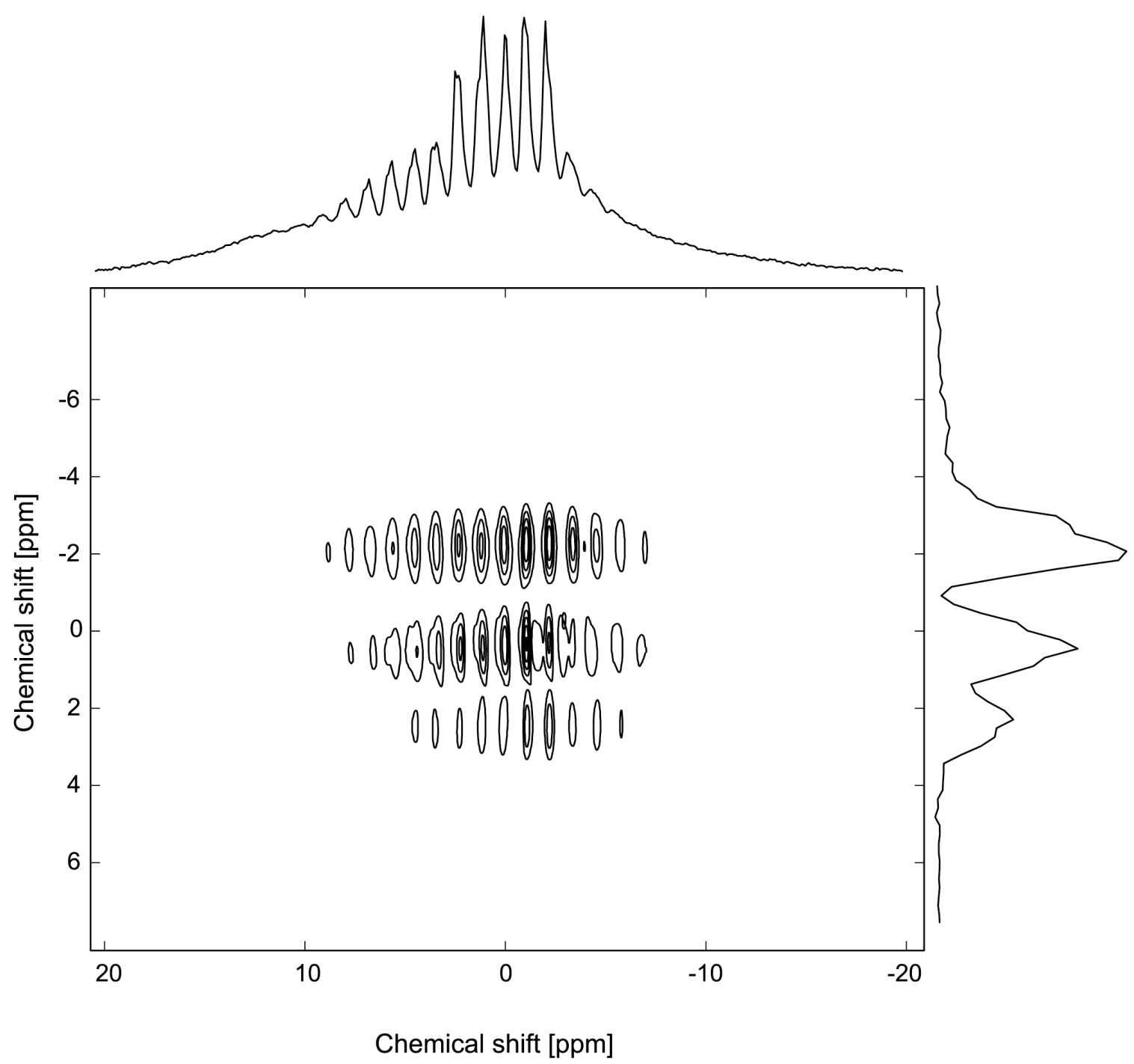




\section{References}

${ }^{1}$ M.D. Hürlimann, D.D. Griffin, J. Mag. Res. 143 (2000) 120.

${ }^{2}$ B. Blümich, P. Blümler, G. Eidmann, A.Guthausen, R. Haken, U. Schmitz, K. Saito, G. Zimmer, Mag. Res. Imag. 16 (1998) 479.

${ }^{3}$ A. Scharfenecker, I. Ardelean, R. Kimmich, J. Mag. Res. 148 (2001) 363.

${ }^{4}$ R.L. Kleinberg, A. Sezginer, D.D. Griffin, M. Fukuhara, J. Mag. Res. 97 (1992) 466.

${ }^{5}$ C.A. Meriles, D. Sakellariou, H. Heise, A. Moulé, A. Pines, Science 293 (2001) 82.

${ }^{6}$ E. R. Andrew, R.G. Eades, Disc. Farad. Soc. 34 (1962) 38.

${ }^{7}$ A. Llor, Z. Olejniczak, A. Pines, J. Chem. Phys. 103 (1995) 3982.

${ }^{8}$ W.T. Dixon, J. Schaefer, M.D. Sefcik, E.O. Stejskal, R.A. McKay, J. Magn. Reson. 49 (1982) 341.

${ }^{9}$ N. Szeverenyi, A. Bax, G.E. Maciel, J. Magn. Reson. 61 (1985) 440.

${ }^{10}$ Z. Gan, J. Am. Chem. Soc., 114 (1992) 8307.

11 J.Z. Hu, D.W. Alderman, C. Ye, R.J. Pugmire, D.M. Grant, J. Magn. Reson. 105 (1993) 82.

12 T.M. de Swiet, M. Tomaselli, M. Hürlimann, A Pines, J. Magn. Reson. 133 (1998) 385.

${ }^{13}$ W.E. Maas, F.H. Laukien, D.J. Cory, J. Am. Chem. Soc. 118 (1996) 13085.

${ }^{14}$ S. Wimperis, J. Magn. Reson. A 109 (1994) 221. 